\title{
Analysis of Timing Jitter in Ring Oscillators Due to Power Supply Noise
}

\author{
T. Pialis and K. Phang \\ pialis@snowbush.com,kphang@eecg.toronto.edu \\ Edward S. Rogers Sr. Dept. of Electrical and Computer Engineering, University of Toronto \\ Toronto, Ontario, M5S 3G4, CANADA
}

\begin{abstract}
This paper presents a time-domain method for estimating the jitter in ring oscillators that is due to power supply noise. The method is used to analyze and compare the RMS cycle-to-cycle jitter of ring oscillators constructed from three possible delay elements: a CMOS digital inverter, a differential pair, and a current steering logic (CSL) inverter. Spice simulations verify the analysis method, and the results indicate that both the differential pair and CSL inverter provide superior supply noise immunity to the CMOS digital inverter.
\end{abstract}

\section{INTRODUCTION}

Ring-based voltage-controlled oscillators (VCO) are well-suited for integration since they require no external components. While their intrinsic phase noise is relatively high compared to that of harmonic or LC oscillators, the dominant noise source is often actually due to the power supply. Such noise typically appears as steps or impulses on the power supply of the oscillator, and it affects both the frequency and phase of the VCO, causing cycle-to-cycle jitter.

Since jitter is a time-domain characteristic, we will use the linear, time-invariant, time-domain oscillator model [1], shown in Figure 1 for a 3 -stage ring oscillator, in our analysis of jitter due to supply noise.

In Figure 1, each element in the ring oscillator is modeled as a cascade of an integrator and a Schmitt trigger block. The output waveforms of the proposed, three-stage ring oscillator are shown in Figure 2. Each inverting stage in the ring contributes a time delay to the total period of oscillation. The delays contributed by the i-th stage, $\tau_{\mathrm{ri}}$ and $\tau_{\mathrm{fi}}$ are measured from the time the output begins switching to the time, $t_{\mathrm{SP}}$, when it reaches the switching threshold voltage $\left(\mathrm{V}_{\mathrm{SP}}\right)$ of the input to the next stage, respectively.

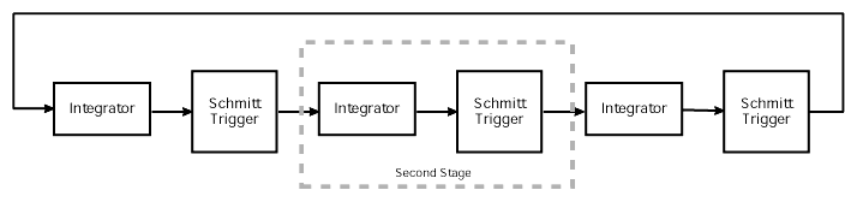

Figure 1: Time Domain Model for a 3-Stage Ring Oscillator

Hence the period of oscillation, $\mathrm{T}_{0}$, for an $\mathrm{N}$-stage ring oscillator is given as:

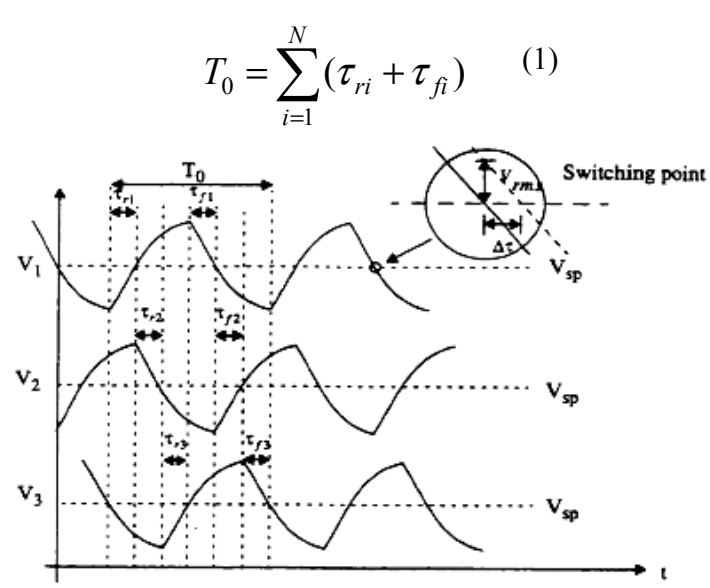

Figure 2: Output Waveforms for the 3-Stage Ring Oscillator

As can be seen in Figure 2, any perturbation voltage on the output of the integrator waveforms alters the time taken to reach the switching threshold. This timing error passes to the other stages in the oscillator ring and contributes to the total output jitter. As shown in [2], an RMS noise voltage $\mathrm{v}_{\mathrm{RMS}}\left(\mathrm{t}_{\mathrm{s}}\right)$, at the time of the threshold crossing, causes timing jitter which is proportional to the voltage error divided by the rising slope $\left(\mathrm{S}_{\mathrm{ri}}\right)$ or falling slope $\left(\mathrm{S}_{\mathrm{fi}}\right)$ of the output waveform. Thus the RMS cycle-to-cycle jitter for an $\mathrm{N}$-stage ring oscillator can be expressed as [1]:

$$
\sigma=\sqrt{\sum_{i=1}^{N}\left[\left(\frac{\Delta V_{O}\left(t_{s p-R}\right)}{S_{r i}}\right)^{2}+\left(\frac{\Delta V_{O}\left(t_{s p-F}\right)}{S_{f i}}\right)^{2}\right]}
$$

To determine the effect of supply noise on the oscillator, the following relation can be used to estimate the change of period due to a small variation in the supply voltage:

$$
\frac{\Delta \mathrm{T}_{\mathrm{OSC}}}{\Delta \mathrm{V}_{\mathrm{DD}}}=2 \mathrm{~N} \cdot \frac{\left.\frac{\Delta \mathrm{V}_{\mathrm{O}}}{\Delta \mathrm{V}_{\mathrm{DD}}}\right|_{\mathrm{V}_{\mathrm{O}}=\mathrm{V}_{\mathrm{SP}}}}{\left.\frac{\Delta \mathrm{V}_{\mathrm{O}}}{\Delta \mathrm{t}}\right|_{\mathrm{V}_{\mathrm{O}}=\mathrm{V}_{\mathrm{SP}}}}
$$

From Equation 3, we see that jitter is essentially dictated by two characteristics of the delay element: the power supply rejection ratio (PSRR), represented by the numerator, and the maximum slope at the switching-point of each delay element, represented by the denominator. In the next two sections, we will derive 
analytic expressions for these two characteristics for the three delay element types shown in Figure 3: a standard CMOS digital inverter, the differential-pair, and the current steering logic (CSL) inverter [3]. $\mathrm{M}$ for the CSL inverter refers to the relative aspect ratio between the input common-source stage device and the diode-connected load device.

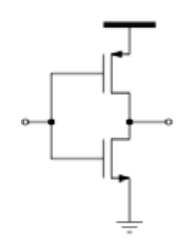

CMOS Digital Inverter

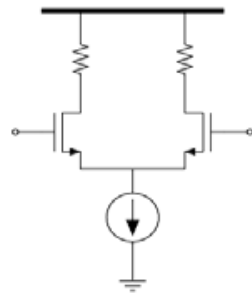

Differential-Pair

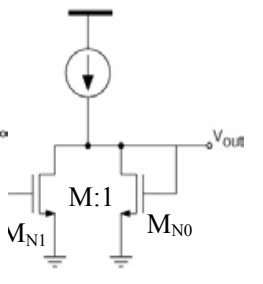

CSL Inverter

Figure 3: Three types of VCO Delay Elements

\section{Mathematical Jitter Analysis}

\subsection{Power Supply Rejection Ratio (PSRR)}

Figures 4(a-c) show the small-signal models for the three delay element types. These models are extracted at the switching point of the circuits, the point at which oscillators are most sensitive to power supply-induced timing jitter [4]. For the small-signal models, $\mathrm{R}_{\mathrm{CS}}$ represents the equivalent output impedance of the cascode current source, and $\mathrm{C}_{\mathrm{P}}$ represents the parasitic capacitance, referenced to VDD, at the output node. This capacitance is the sum of stray drain-bulk and drain-gate capacitances from the cascode transistor of the PMOS current source.

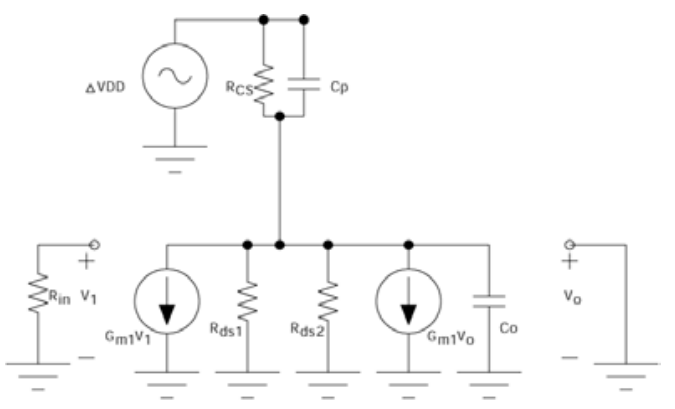

Figure 4a): Equivalent Small-Signal Circuit for the CSL Inverter

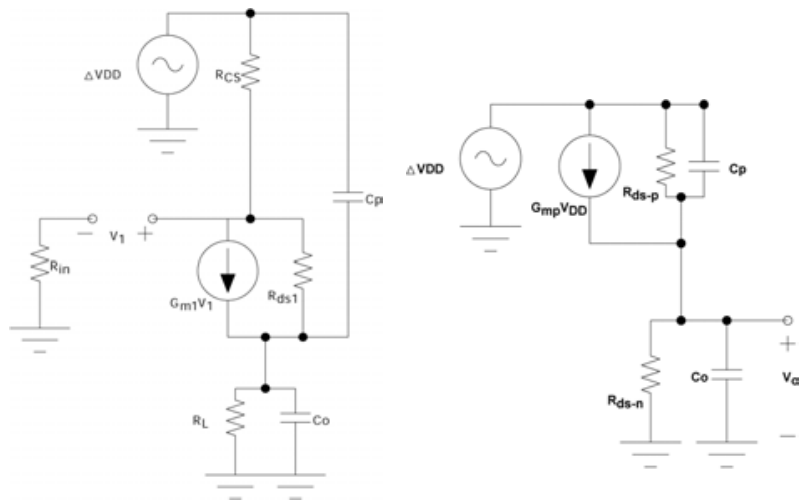

Figure 4b): Equivalent Small- Figure 4c): Equivalent SmallSignal Circuit for the Diff. Pair Signal Circuit for the CMOS Digital Inverter
Table 1 summarizes the transfer functions derived from Figures 4(a-c). These equations were compared with the Spice simulation results obtained using a $0.18 \mu$ digital CMOS technology and which are shown in Figure 5.

\begin{tabular}{|c|c|}
\hline Element Type & $\operatorname{PSRR}\left(\Delta \mathbf{V}_{\mathrm{o}} / \Delta \mathbf{V}_{\mathrm{DD}}\right)(\mathrm{V} / \mathrm{V})$ \\
\hline $\begin{array}{c}\text { Digital } \\
\text { Inverter }\end{array}$ & $\frac{\Delta \mathrm{V}_{\mathrm{O}-\mathrm{INV}}(\mathrm{s})}{\Delta \mathrm{V}_{\mathrm{DD}}(\mathrm{s})} \approx \frac{\mathrm{g}_{\mathrm{m}}+\mathrm{C}_{\mathrm{P}} \cdot \mathrm{s}}{2 \mathrm{~g}_{\mathrm{ds}}+\mathrm{s} \cdot\left(\mathrm{C}_{\mathrm{p}}+\mathrm{C}_{\mathrm{O}}\right)}$ \\
\hline $\begin{array}{c}\text { Differential } \\
\text { Pair }\end{array}$ & $\frac{\Delta \mathrm{V}_{\mathrm{O}-\mathrm{DIFF}}(\mathrm{s})}{\Delta \mathrm{V}_{\mathrm{DD}}(\mathrm{s})} \approx \frac{1+\mathrm{g}_{\mathrm{m}} \mathrm{r}_{\mathrm{ds}}{ }^{2} \mathrm{C}_{\mathrm{P}} \cdot \mathrm{s}}{\mathrm{g}_{\mathrm{m} 2}{ }^{2} \mathrm{r}_{\mathrm{ds}}{ }^{2}+\mathrm{s} \cdot \mathrm{g}_{\mathrm{m}} \mathrm{r}_{\mathrm{ds}}{ }^{2}\left(\mathrm{C}_{\mathrm{p}}+\mathrm{C}_{\mathrm{O}}\right)}(5)$ \\
\hline CSL Inverter & $\frac{\Delta \mathrm{V}_{\mathrm{O}-\mathrm{CSL}}(\mathrm{s})}{\Delta \mathrm{V}_{\mathrm{DD}}(\mathrm{s})} \approx \frac{1+\mathrm{g}_{\mathrm{m}} \mathrm{r}_{\mathrm{ds}}{ }^{2} \mathrm{C}_{\mathrm{P}} \cdot \mathrm{s}}{\mathrm{g}_{\mathrm{m} 2}{ }^{2} \mathrm{r}_{\mathrm{ds}}{ }^{2}+\mathrm{s} \cdot \mathrm{g}_{\mathrm{m}} \mathrm{r}_{\mathrm{ds}}{ }^{2}\left(\mathrm{C}_{\mathrm{p}}+\mathrm{C}_{\mathrm{O}}\right)}(6)$ \\
\hline
\end{tabular}

Table 1: Analytic Expressions for the PSRR of Three Delay Element Types

We draw the following observations:

1) The PSRR for the CMOS digital inverter is low-pass in nature, flattening out at high-frequencies. The digital inverter exhibits extremely poor low-frequency PSRR compared to either of the other two delay elements since any power-supply noise is amplified by the small-signal gain of the inverter. This is one of the reasons why digital inverters are not used in VCOs that are intended for jittersensitive applications.

2) The PSRR of both the differential-pair and CSL inverter are nearly identical over all frequency bands. The PSRR is approximately $1 /\left(\mathrm{g}_{\mathrm{m}} \cdot \mathrm{r}_{\mathrm{ds}}\right)^{2}$ at low frequencies, gradually degrading at higher frequencies until it reaches $\mathrm{C}_{\mathrm{p}} /\left(\mathrm{C}_{\mathrm{p}}+\mathrm{C}_{\mathrm{O}}\right)$, at which point the circuit operates as a capacitive voltage divider between the stray capacitances to VDD, $\mathrm{C}_{\mathrm{p}}$, and the dominant load capacitance to VSS, $\mathrm{C}_{\mathrm{O}}$.

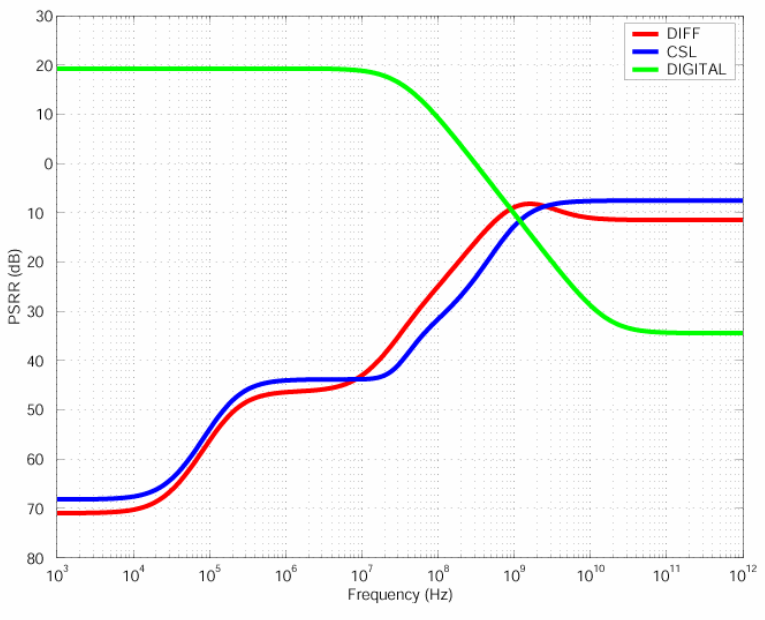

Figure 5: Spice Simulation Results Showing the PSRR for all 3 Types of Delay Cells 


\subsection{Rising and Falling Slopes}

Expressions for the rising and falling slopes for both the CMOS digital inverter and the differential-pair can be found in references [1] and [5] respectively, while the derivation for the CSL inverter can be found in the Appendix. Table 2 summarizes the results for the three delay elements for an N-stage oscillator.

\begin{tabular}{|c|c|c|}
\hline Element & \\
\hline $\begin{array}{c}\text { Digital } \\
\text { Inverter [1] }\end{array}$ & $\frac{\beta \mathrm{p} \cdot \mathrm{V}_{\mathrm{DD}}^{2}}{2 \mathrm{C}_{\mathrm{L}}} \cdot\left(\frac{\pi}{2}-0.35\right)$ & $-\frac{\beta \mathrm{n} \cdot \mathrm{V}_{\mathrm{DD}}^{2}}{2 \mathrm{C}_{\mathrm{L}}} \cdot\left(\frac{\pi}{2}-0.35\right)$ \\
\hline $\begin{array}{c}\text { Differential } \\
\text { Pair [5] }\end{array}$ & $\frac{\beta_{0} \cdot \mathrm{V}_{\mathrm{EFF}-0}{ }^{2}}{2 \mathrm{C}_{\mathrm{L}}}$ & $-\frac{\beta_{0} \cdot \mathrm{V}_{\mathrm{EFF}-0}{ }^{2}}{2 \mathrm{C}_{\mathrm{L}}}$ \\
\hline $\begin{array}{c}\text { CSL } \\
\text { Inverter } \\
\text { (Appendix) }\end{array}$ & $\frac{\beta_{0} \cdot \mathrm{V}_{\mathrm{EFF}-0}{ }^{2}}{2 \mathrm{C}_{\mathrm{L}}} \cdot \frac{\mathrm{M}}{(\mathrm{M}+1)}$ & $-\frac{\beta_{0} \cdot \mathrm{V}_{\mathrm{EFF}-0}{ }^{2}}{2 \mathrm{C}_{\mathrm{L}}} \cdot \frac{\mathrm{M}^{2}}{(\mathrm{M}+1)}$ \\
\hline
\end{tabular}

Table 2: Rise and Fall Slopes

Here, $\mathrm{V}_{\text {eff- } 0}$ is the gate overdrive voltage present when the delay elements are at their switching threshold, $C_{L}$ represents the total lumped output load capacitance of each delay stage, and $\beta_{0}, \beta_{\mathrm{p}}$ and $\beta_{\mathrm{n}}$ represent the $\mu \cdot \mathrm{C}_{\mathrm{ox}} \cdot \mathrm{W} / \mathrm{L}$ of the analog NMOS input transistors along with the digital inverter $\mathrm{p}$ - and $\mathrm{n}$-channel devices, respectively.

\section{Analytical and Simulation Results}

Having derived analytic expressions for both the PSRR and the rising and falling slopes for each delay element type, we can now estimate the frequency sensitivity to power supply noise using Equation 3. Assuming the supply voltage is modulated by a sinusoid, $\Delta V_{D D}=V_{m} \cdot \cos \left(\omega_{m} t\right)$, the oscillator period deviations, $\Delta \mathrm{T}$, can be derived for all three delay elements. Subsequently, the autocorrelation functions for the oscillator period deviations, $\Delta \mathrm{T}$, with respect to $t$ can be obtained using the following relation:

$$
\mathrm{C}_{\mathrm{TT}}(\tau)=E[\Delta \mathrm{T}(\mathrm{t}+\tau) \cdot \Delta \mathrm{T}(\mathrm{t})]
$$

Subsequently, it has been proven in [6] that the mean square cycle-to-cycle jitter $\sigma^{2}$ is equal to:

$$
\sigma^{2}=\left.2 \mathrm{C}_{\mathrm{TT}}(\tau)\right|_{\tau=0}-\left.2 \mathrm{C}_{\mathrm{TT}}(\tau)\right|_{\tau=1 / f_{0}}
$$

Thus, from Equations 7 and 8, the RMS cycle-to-cycle jitter due to power supply noise can be estimated for all three types of oscillator rings. Table 3 lists the final results:

\begin{tabular}{|c|c|}
\hline Element & Estimated, RMS Cycle-to-Cycle Jitter: $\Delta \mathbf{T}_{\mathbf{C C}}$ \\
\hline $\begin{array}{c}\text { Digital } \\
\text { Inverter }\end{array}$ & $\frac{2 \mathrm{NC}_{\mathrm{L}}}{\beta_{0} \cdot \mathrm{V}_{\mathrm{DD}}{ }^{2} \cdot k_{I N V-2}} \cdot \sqrt{\frac{\mathrm{g}_{\mathrm{m}}{ }^{2}+\left(\mathrm{C}_{\mathrm{p}} \omega_{\mathrm{m}}\right)^{2}}{4 \mathrm{~g}_{\mathrm{ds}}{ }^{2}+\left(\mathrm{C}_{\mathrm{p}}+\mathrm{C}_{\mathrm{O}}\right)^{2} \cdot \omega_{\mathrm{m}}{ }^{2}}} \cdot V_{m} \cdot \sqrt{1-\cos \left(2 \pi \frac{f_{m}}{f_{O}}\right)}$ \\
$(9)$
\end{tabular}

Table 3: Table of $\sigma_{\text {RMS }}$, Estimated RMS Cycle-to-Cycle Jitter Due to Supply Noise
Spice simulations were performed to verify the RMS cycle-tocycle jitter values estimated by Equations 9 to 11 . Figure 6 shows the output spectrum and measured RMS cycle-to-cycle jitter for a 5-stage ring oscillator implemented utilizing each of the three delay element types. All of the oscillator rings were tuned to have a center frequency of $129 \mathrm{MHz}$. The $1.8 \mathrm{~V}$ supply was modulated with a $10 \%$ sinusoid at frequencies of $10 \mathrm{MHz}$, $88 \mathrm{MHz}, 129 \mathrm{MHz}$ and $188 \mathrm{MHz}$. Figure 6 shows the output spectrum for the $10 \mathrm{MHz}$ case, in which the tone introduced by the power supply noise is modulated by the spectrum of the oscillator ring itself, introducing distortion and timing jitter in the output spectrum.

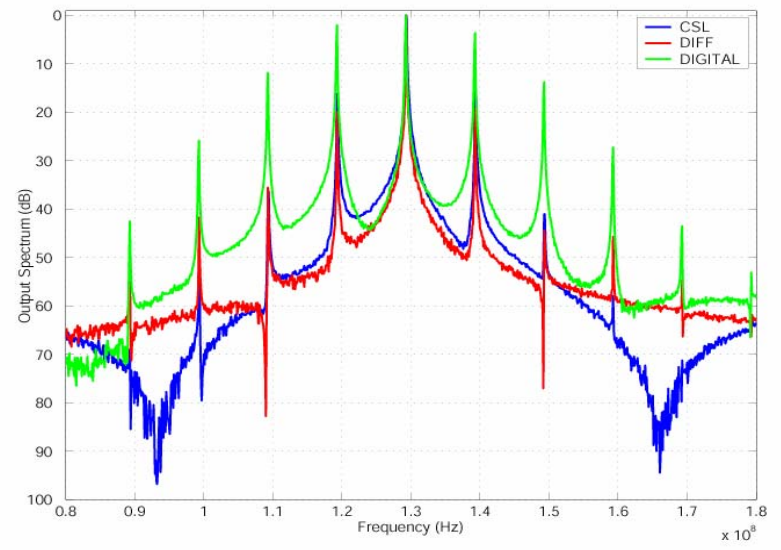

Figure 6: Output Spectrums, $\mathrm{F}_{\mathrm{M}}=10 \mathrm{MHz}$

Table 4 places the simulated results alongside the estimated values from our analysis. Table 4 compares estimated and simulated data for both the CSL and differential-pair delay elements. Both the differential pair and the CSL inverter analysis and simulations exhibited the best matching since the linear, time-invariant model of an oscillator ring used in this analysis is most accurate for oscillators with small-signal voltage swings [7]. In addition, simulation results also verified the relation between timing jitter and the spectral makeup of injected supply and substrate noise relative to the oscillator frequency, as predicted by Equations 9-11.

\begin{tabular}{|c|c|c|c|c|}
\hline $\begin{array}{c}\text { RMS } \\
\text { Jitter }\end{array}$ & Result & $\begin{array}{c}\text { Digital } \\
\text { Inverter }\end{array}$ & $\begin{array}{c}\text { Diff. } \\
\text { Pair }\end{array}$ & $\begin{array}{c}\text { CSL } \\
\text { Inverter }\end{array}$ \\
\hline $\begin{array}{c}\boldsymbol{\sigma}_{\text {RMs }}: \\
\mathbf{F}_{\mathbf{M}}=\mathbf{1 0 M h z}\end{array}$ & Calculated & $873 \mathrm{ps}$ & $42 \mathrm{ps}$ & $24 \mathrm{ps}$ \\
\cline { 2 - 5 } & Simulated & $373 \mathrm{ps}$ & $94 \mathrm{ps}$ & $84 \mathrm{ps}$ \\
\hline $\begin{array}{c}\boldsymbol{\sigma}_{\text {RMs }}: \\
\mathbf{F}_{\mathbf{M}}=\mathbf{8 8 M h z}\end{array}$ & Calculated & $1.59 \mathrm{~ns}$ & $153 \mathrm{ps}$ & $36 \mathrm{ps}$ \\
\cline { 2 - 5 } & Simulated & $517 \mathrm{ps}$ & $157 \mathrm{ps}$ & $92 \mathrm{ps}$ \\
\hline $\begin{array}{c}\boldsymbol{\sigma}_{\mathrm{RMS}}: \\
\mathbf{F}_{\mathbf{M}}=\mathbf{1 2 9 M h z}\end{array}$ & Calculated & $0 \mathrm{ps}$ & $0 \mathrm{ps}$ & $0 \mathrm{ps}$ \\
\cline { 2 - 5 } & Simulated & $2.4 \mathrm{ps}$ & $2.8 \mathrm{ps}$ & $2.1 \mathrm{ps}$ \\
\hline $\begin{array}{c}\boldsymbol{\sigma}_{\mathrm{RMS}}: \\
\mathbf{F}_{\mathbf{M}}=\mathbf{1 8 8 M h z}\end{array}$ & Calculated & $391 \mathrm{ps}$ & $141 \mathrm{ps}$ & $30 \mathrm{ps}$ \\
\cline { 2 - 5 } & Simulated & $105 \mathrm{ps}$ & $135 \mathrm{ps}$ & $49 \mathrm{ps}$ \\
\hline
\end{tabular}

Table 4: Table of $\sigma_{\text {RMS }}$, Estimated and Simulated RMS Cycle-toCycle Jitter for Various Power-Supply Modulation Frequencies, $\mathrm{F}_{\mathrm{M}}=10 \mathrm{MHz}, 88 \mathrm{MHz}, 129 \mathrm{MHz}$ and $188 \mathrm{MHz}$ 
Figure 7 illustrates how the final analytic expressions in Table 3 can be used to predict the sensitivity of the ring oscillator to the frequency spectrum of the supply noise. This is critical in understanding what bands of power-supply and substrate noise should be isolated from oscillator rings to achieve low jitter performance. Figure 7 predicts that both the CSL inverter and differential-pair based ring oscillators exhibit excellent lowfrequency power rejection far superior to the digital inverter based oscillator due to the high PSRR of both cascoded delay elements at low-frequencies. However, at higher frequencies, the reduced PSRR of both the differential and the CSL oscillator rings cause both of their predicted RMS, cycle-cycle jitter transfer-functions to increase significantly. Furthermore, Equations 9 to 11 predict that all three oscillator rings should exhibit nulls in their jitter transfer-functions at the harmonics of the oscillator ring center-frequency, (i.e., for $f_{m}=f_{O}, 2 \cdot f_{O}, 3 \cdot f_{O}$, etc.); indeed, the simulation results in Table 4 for $f_{m}=f_{O}$ validate this prediction.

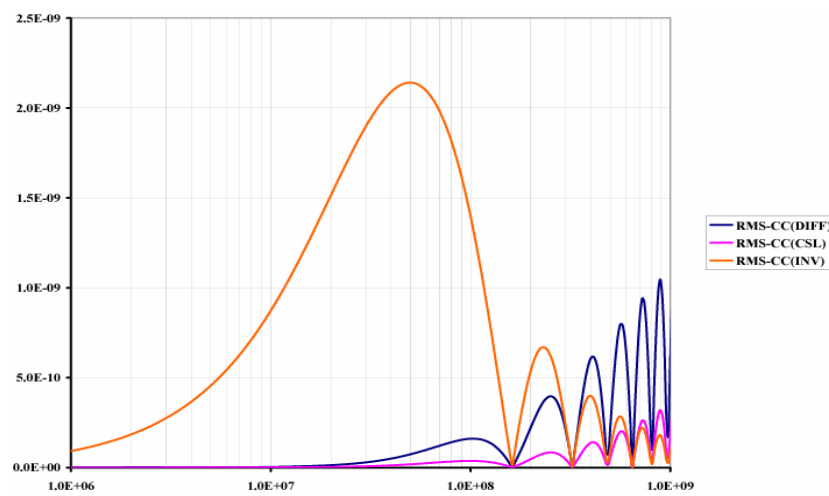

Figure 7: Plot of Calculated $\sigma_{\text {RMS }}$ Versus Supply Noise Frequency, $\mathrm{F}_{\mathrm{M}}$,

\section{Conclusions}

The equations developed in this work can significantly aid in the design of ring oscillators. Complex relationships such as timing jitter are very difficult and time-consuming to simulate and model, so these expressions provide an apriori means of estimating the RMS cycle-to-cycle jitter due to power supply noise. The proposed analysis method allows critical design parameters to be estimated early in the design stage, allowing approximate sizings of transistors and phase-locked loop parameters to be optimized, thereby reducing the time required to iterate and optimize both the design of the oscillator and of the complete phase-locked loop.

\section{REFERENCES}

[1] Lizhong Sun. High-Speed Submicron CMOS Oscillators and PLL Clock Generators. M.Eng. Dissertation, Carleton University, Ottawa, April 1999.

[2] A. A. Abidi and R. G. Meyer, "Noise in Relaxation Oscillators," IEEE J. Solid-State Circuits, Vol.18, pp.794-802, December 1983.
[3] D. Allstot, G. Lang and H. Yang, "Current-Mode Logic Techniques for CMOS Mixed-Mode ASICs," Proc. IEEE Custom Integrated Circuits Conf., 1991, pp.25.2.1-25.2.4.

[4] A. Hajimiri and T.Lee, "A General Theory of Phase Noise in Electrical Oscillators, "JSSC, Feb. 1998.

[5] John McNeill, "Jitter in Ring Oscillators," IEEE J. SolidState Circuits, Vol.32, pp.870-879, June 1997.

[6] F. Herzel and B. Razavi, "Oscillator Jitter Due to Supply and Substrate Noise, Proc. CICC, pp.489-492, May 1998.

[7] Ali Hajimiri. Jitter and Phase Noise in Electrical Oscillators. Dissertation, Stanford University, Palo Alto California, November 1998.

\section{Appendix: Rising and Falling Slopes of a CSL Inverter}

Referring to Figure 3, when a positive input step voltage is applied to the CSL inverter, at the switching threshold, both $\mathrm{M}_{\mathrm{N} 0}$ and $\mathrm{M}_{\mathrm{N} 1}$ operate in the active region. As a result, performing $\mathrm{KCL}$ at the output node results in:

$$
\mathrm{I}_{\mathrm{CS}}=\frac{\beta_{0}}{2} \cdot\left(\mathrm{V}_{\mathrm{O}}-\mathrm{V}_{\mathrm{TN}}\right)^{2}+\mathrm{M} \cdot \mathrm{I}_{\mathrm{CS}}+\mathrm{C}_{\mathrm{L}} \frac{\mathrm{dV}_{\mathrm{O}}}{\mathrm{dt}}
$$

It can also be shown that the switching point $\left(\mathrm{V}_{\mathrm{SP}}\right)$ for a CSL inverter biased with a tail current of $\mathrm{I}_{\mathrm{CS}}$ is:

$$
\mathrm{V}_{\mathrm{sp}}=\mathrm{V}_{\mathrm{TN}}+\sqrt{\frac{2 \mathrm{I}_{\mathrm{CS}}}{(\mathrm{M}+1) \beta_{0}}}
$$

As a result, the falling slope of a CSL inverter at the switching point can be found by substituting Equation A-2 into Equation A-1, yielding:

$$
{\frac{\mathrm{dV}}{\mathrm{d} \mathrm{V}_{\text {-fall }}}}=-\frac{\beta_{0} \cdot \mathrm{V}_{\mathrm{EFF}-0}{ }^{2}}{\mathrm{C}_{\mathrm{L}}} \cdot \frac{\mathrm{M}^{2}}{2 \cdot(\mathrm{M}+1)}
$$

Similarly, to calculate the rising slope of the CSL inverter, it is now assumed a negative input step voltage is applied to the CSL inverter. At the switching threshold, $\mathrm{V}_{\mathrm{O}}=\mathrm{V}_{\mathrm{SP}}$ and only $\mathrm{M}_{\mathrm{N} 0}$ is on and operating in the active region. As a result, this time performing $\mathrm{KCL}$ at the output node results in:

$$
\mathrm{I}_{\mathrm{CS}}=\frac{\beta_{0}}{2} \cdot\left(\mathrm{V}_{\mathrm{O}}-\mathrm{V}_{\mathrm{TN}}\right)^{2}+\mathrm{C}_{\mathrm{L}} \frac{\mathrm{dV}}{\mathrm{dt}}
$$

Once again, Equation A-2 is substituted, yielding a rising slope of:

$$
\frac{\mathrm{dV}_{\mathrm{O}}}{\mathrm{dt}} \text {-rise }=\frac{\beta_{0} \cdot \mathrm{V}_{\mathrm{EFF}-0}{ }^{2}}{\mathrm{C}_{\mathrm{L}}} \cdot \frac{\mathrm{M}}{2 \cdot(\mathrm{M}+1)}
$$

As can be seen in Equations A-3 and A-5, the slope during a falling transition is typically $\mathrm{M}$ time larger than for a rising transition because of the large, transient, charging current provided by the switching transistor $\mathrm{M}_{\mathrm{N} 1}$, which is on for falling transitions only. 